\title{
Author Correction: RNA interference-independent reprogramming of DNA methylation in Arabidopsis
}

Taiko Kim To (D), Yuichiro Nishizawa, Soichi Inagaki, Yoshiaki Tarutani, Sayaka Tominaga, Atsushi Toyoda (D, Asao Fujiyama, Frédéric Berger (iD) and Tetsuji Kakutani (i)

Correction to: Nature Plants https://doi.org/10.1038/s41477-020-00810-z, published online 30 November 2020.

In the version of this Article originally published, panels $\mathrm{d}-\mathrm{h}$ were mistakenly omitted from Extended Data Fig. 9. This has been corrected and the revised figure is available online.

Published online: 16 December 2020

https://doi.org/10.1038/s41477-020-00839-0

(c) The Author(s), under exclusive licence to Springer Nature Limited 2020 